\title{
Contribution à l'étude sérologique et physio-pathologique de la streptothricose bovine
}

\author{
par R. GAULIER (*), J. M. BLANCOU $(* *)$, P. BOURDIN $(* * *)$, \\ J. J. RIBOT $\left({ }^{*}\right)$, J. RAMISSE $(* *)$, H. SERRES $(* *)$
}

(avec la collaboration technique de Mme F. ALEXANDRE)

\begin{abstract}
RESUME
L'étude biochimique du sang de bovins atteints de streptothricose révèle d'importantes modifications de sa composition, en particulier une augmentation du fibrinogène, une baisse du cholestérol, un abaissement du rapport $\mathrm{Ca} / \mathrm{P}$ et de profonds bouleversements de ses constituants protidiques: ces modifications suivent étroitement l'évolution de la maladie et peuvent permettre d'en établir le pronostic.

L'étude sérologique démontre également une concordance entre l'évolution des lésions et le taux d'anticorps circulants.

L'étude histopathologique des organes de bovins morts de la maladie naturelle révèle deux types de lésions principales: inflammatoires (dues à l'action directe de la bactérie sur la peau, la rate et les ganglions) et toxiques, localisées au fole et au rein.
\end{abstract}

\section{INTRODUCTION}

Lors de l'évolution des formes graves de streptothricose chez les bovins, il apparaît que l'état général des animaux va en s'affaiblissant au cours de la maladie. Cet affaiblissement aboutit généralement à la mort du sujet si les lésions cutanées ne rétrocèdent pas. Une atteinte générale grave de l'organisme est toujours reflétée par des modifications de la physiologie de l'individu (constantes biologiques, réactions de défense immunitaire) et de l'intégrité de ses organes. C'est pourquoi cette étude a été entreprise dans le but de définir les modifications physiopathologiques les plus significatives de la streptothricose bovine.

Les recherches ont été menées sur des animaux vivant à Madagascar, atteints de

(") Pharmacien-Chimiste en Chef de 2e Classe.

(**) I.E.M.V.T., Laboratoire Central de l'Elevage, B.P. $n^{\circ} 862$, Tananarive.

(***) I.E.M.V.T., Laboratoire National de l'Elevage, B.P. no 2057, Dakar-Hann. maladie naturelle, dont les constantes biologiques et les réactions immunitaires ont été comparées à celles d'animaux sains vivant dans les mêmes conditions. En cas de maladie grave, entraînant la mort, il a été pratiqué une étude histopathologique des principaux organes du cadavre. L'ensemble de l'étude porte sur 261 prélèvements, effectués sur 111 animaux différents, dont les caractéristiques principales sont détaillées dans le tableau I :

Les animaux de moins de 2 ans ont été contaminés lors de leur élevage en plein air: ils ont été alors rentrés à l'étable pour les besoins de l'expérience. Les animaux de plus de 2 ans vivaient toujours en élevage extensif de plein air.

Les méthodes employées pour les différentes analyses effectuées chez ces animaux, ainsi que les résultats obtenus, sont exposés dans l'ordre suivant :

1. Etude comparée des constituants du sang de bovins atteints ou non de streptothricose. 
TABLEAU N ${ }^{\circ} \mathrm{I}$

\begin{tabular}{|c|c|c|c|c|}
\hline & $\mathrm{R} a \mathrm{c} e$ & $S e x e$ & A $g e$ & $\begin{array}{c}\text { Etat des lésions } \\
\text { cutanées }\end{array}$ \\
\hline $\begin{array}{l}\text { Animaux } \\
\text { atteints de } \\
\text { Streptothricose }\end{array}$ & $\begin{array}{l}\text { Zëbus malgaches } \\
\text { ou croisës de } \\
\text { zëbus Brahman }\end{array}$ & $\begin{array}{l}31 \text { femelles } \\
31 \text { mâles }\end{array}$ & $\begin{array}{l}27 \text { sujets de moins } \\
\text { de } 2 \text { ans } \\
18 \text { sujets de } 4 \text { à } 6 \text { ans } \\
17 \text { sujets de plus de } \\
6 \text { ans. }\end{array}$ & $\begin{array}{r}+++31 \\
++: 16 \\
+: 15\end{array}$ \\
\hline \multirow{2}{*}{$\begin{array}{l}\text { Animaux } \\
\text { têmoins }\end{array}$} & $\begin{array}{l}\text { Zêbus malgacines } \\
\text { ou croisés de } \\
\text { zêbus Brahman }\end{array}$ & $\begin{array}{l}31 \text { femelles } \\
16 \text { mâles }\end{array}$ & $\begin{array}{l}20 \text { sujets de } 4 \text { à } 6 \text { ans } \\
27 \text { sujets de plus de } \\
6 \text { ans. }\end{array}$ & $\begin{array}{l}\text { Pas de lësions } \\
\text { cutanées visibles } \\
\text { à l'oeil nu }\end{array}$ \\
\hline & $\begin{array}{l}\text { Zëbus malgaches } \\
\text { croisés de } \\
\text { taurins }\end{array}$ & 2 femelles & $\begin{array}{l}\text { Sujets de plus de } \\
6 \text { ans. }\end{array}$ & \\
\hline
\end{tabular}

+++ = atteinte gënéralisẻe; ++ = atteinte nette (plus de la moitié du corps); + = atteinte lêgère.

2. Etude de l'évolution des réactions sérologiques au cours de la streptothricose naturelle.

3. Etude histologique des lésions organiques dans la streptothricose.

\section{ETUDE COMPAREE \\ DE CONSTITUANTS DU SANG \\ DE BOVINS EXEMPTS \\ DE STREPTOTHRICOSE ET DE BOVINS \\ ATTEINTS DE STREPTOTHRICOSE}

\section{METHODES DE DETERMINATION}

Urée: Méthode colorimétrique au paradiméthyl-amino-benzaldéhyde (2). Cholestérol total: Dosage photométrique par la réaction de LIEBERMANN, selon la technique de RAPPOPORT et EICHHORN (10). Glucose : Méthode à l'ortho-toluidine de FINETTI et BUFFARD (7). Protéines sériques: Méthode au biuret de RIEGLER, technique de CORNALL (8). Fibrinogène: Microdosage photométrique par la technique de LECLERC et KHODABANDEH (8). Phosphore minéral: Technique de BRIGGS (8). Calcium: Microdosage par complexométrie (méthode à la calcéine) (8). Cholinestérases: Méthode électro-pH métrique de CHARY, JAYOT et BOCQUET (5). Bilirubine totale: Diazoréaction d'HIJMANS VAN BERGH, après libération de la bilirubine par réaction de JENDRASSIK et GROF (8). Créatinine: Réaction de JAFFE sur défécat trichloracé- tique du sérum (10). Azote "polypeptidique": Méthode de l'" Index-tyrosine " de GOIFFON et SPAEY (8). Acide urique: Microméthode colorimétrique de FOLIN et DENIS, adaptée par GRIGAUT (8). Hémoglobine: Dosage photométrique après transformation en cyanhémoglobine (4). Phosphatases acides: Selon méthode de BABSON, READ et PHILLIPS (Phosphatabs-acide WARNER-CHILCOTT) (1). Phosphatases alcalines: Selon méthode de KLEIN, BABSON et READ (Phosphatabs-alcaline WARNER-CHILCOTT) (9). Transaminases glutamino-oxaloacétique (T.G.O.) et transaminase glutamino-pyruvique (T.G.P.): D'après technique de FRANKEL et REITMAN (Réactifs HAURY) (16). Protéinogramme: Selon technique de GRASSMAN et HANNIG (Electrophorèse du sérum sur papier. Coloration au Noir Amido 10 B. Interprétation par Integraph ELPHOR) (11).

\section{RESULTATS}

Pour chaque constituant nous n'avons mentionné qu'une valeur par série d'animaux, cette valeur étant représentée par la moyenne des dosages effectués dont nous indiquons par conséquent l'erreur type $(\mathrm{Sm})$ et les valeurs extrêmes (v.e.). La comparaison des moyennes entre les sujets sains et malades a donné lieu à une interprétation statique qui détermine si elles diffèrent de façon significative ou non.

Nous avons étudié deux séries d'animaux : des sujets adultes, tous gravement atteints, et de jeunes sujets dont 8 étaient gravement 
atteints et 14 légèrement. Des témoins ont été choisis parmi des animaux du même âge et vivant dans les mêmes conditions d'élevage (4 jeunes et 32 à 49 adultes). Les constituants pour lesquels ne s'est révélée aucune modification significative au cours de la maladie, sont étudiés les premiers, suivis de ceux pour lesquels apparait une variation significative ou hautement significative.

- Urée :

Bovins adultes. Ches 38 malades (71 dosages) le taux moyen d'urée est de $0,26 \mathrm{~g}$ par 1 $(\mathrm{Sm}=0,010$ - v.e. : $0,10-0,45)$ alors qu'il était de $0,23 \mathrm{~g}$ chez 43 sujets sains $(\mathrm{Sm}=0,010$ - v.e. : 0,10 - 0,37).

Jeunes bovins. Dans les cas graves le taux moyen est de $0,35 \mathrm{~g}(\mathrm{Sm}=0,024$ - v.e.: $0,22-0,50$ ) de même que dans les cas légers $(\mathrm{Sm}=0,010)$. Chez 4 sujets sains il est de $0,32 \mathrm{~g}(\mathrm{Sm}=0,010)$.

Ces différences sont non significatives statistiquement. D'ailleurs un bovin adulte très gravement atteint avait un taux normal de $0,24 \mathrm{~g}$.
- Glucose :

Bovins adultes. Chez 7 malades (35 dosages) le taux moyen est de $0,72 \mathrm{~g}$ par $1(\mathrm{Sm}=0,035)$ et chez 45 sujets sains d'abattoir de $0,77 \mathrm{~g}$ $(\mathrm{Sm}=0,036)$.

Jeunes bovins. Dans les cas graves le taux moyen est de $0,67 \mathrm{~g}(\mathrm{Sm}=0,057)$. Il est de $0,76 \mathrm{~g}(\mathrm{Sm}=0,037)$ dans les cas légers et de $0,72 \mathrm{~g}$ chez 4 sujets sains $(\mathrm{Sm}=0,074)$.

Toutes ces différences sont non significatives statistiquement.

\section{- Protéines sériques totales :}

Bovins adultes. Chez 38 malades (70 dosages) le taux moyen est de $91,7 \mathrm{~g}$ par 1 $(\mathrm{Sm}=1,62)$ et de 93,7 ( $\mathrm{Sm}=1,29) \mathrm{chez}$ 44 témoins sains : différence non significative.

Des écarts importants par rapport à la moyenne existant tant chez les malades (75 à $111 \mathrm{~g}$ ) que chez les témoins (73 à $114 \mathrm{~g}$ ). Alors que ce taux reste relativement stable chez les sujets sains ou peu atteints, il peut varier beaucoup au cours du temps comme l'indiquent les chiffres suivants concernant une vache atteinte de streptothricose généralisée (7 dosages en 5 mois).

\begin{tabular}{|lrrrrrrr|}
\hline Protides (gramme par litre) & 90 & 100 & 97 & 104 & 95 & 91 & 101 \\
Albumines sériques (p. 100) & 29 & 27 & 23 & 23 & 23 & 22 & 19 \\
Gamma-globulines (p. 100) & 40 & 43 & 48 & 46 & 48 & 48 & 55 \\
\hline
\end{tabular}

On constate l'abaissement des albumines et l'augmentation corrélative des gamma-globulines.

Jeunes bovins. Le taux moyen chez 22 malades (61 dosages) est de $79 \mathrm{~g}$ par 1 dans 8 cas graves $(\mathrm{Sm}=2,96)$ et de 76,5 $\mathrm{g}(\mathrm{Sm}=1,87)$ dans les cas légers. Chez les sujets sains : $78,6 \mathrm{~g}$ $(\mathrm{Sm}=5,00)$. Ces chiffres sont donc inférieurs à ceux des adultes mais ne présentent pas de différences notables entre eux. Comme chez les adultes, il n'existe pas de relation entre le taux de protides et le pourcentage d'albumine ou de gamma-globulines sériques.

- Créatinine :

Bovins adultes. Chez 8 malades, la valeur moyenne est de $2 \mathrm{mg}$ par $1(\mathrm{Sm}=0,2$ - v.e. :
$0,6-2,1$ ), soit la même que chez 45 sujets sains $(\operatorname{Sm}=0,1-$ v.e. : $0,8-3,7)$.

Jeunes bovins. Dans les cas graves : $0,9 \mathrm{mg}$ $(\mathrm{Sm}=0,12)$, dans les cas légers : $1 \mathrm{mg}$ $(\mathrm{Sm}=0,05)$ et chez les sujets sains : $0,9 \mathrm{mg}$ $(\mathrm{Sm}=0,07)$.

Ce taux est donc inférieur à celui des adultes mais, comme chez eux, la différence est non significative entre malades et témoins.

\section{- Acide urique :}

Bovins adultes. Chez 13 malades (23 dosages) la teneur moyenne est de $8 \mathrm{mg}$ par 1 $(\mathrm{Sm}=0,6)$ et de $7 \mathrm{mg}(\mathrm{Sm}=0,4)$ chez 32 sujets sains. Toutes ces valeurs étaient groupées entre 5 et $13 \mathrm{mg}$, sauf $18 \mathrm{mg}$ dans un cas très grave. La différence est non significative entre malades et témoins. 
Jeunes bovins. Le taux moyen chez 22 malades (63 dosages) est de $8 \mathrm{mg}$ par 1 dans les cas graves $(\mathrm{Sm}=0,5)$ et les cas légers ( $\mathrm{Sm}=0,3$ ), de même que chez les sujets sains $(\mathrm{Sm}=1,2)$ avec des valeurs extrêmes de 3 et $13 \mathrm{mg}$. Ceci confirme les résultats relevés chez les adultes.

\section{- Hémoglobine :}

Bovins adultes. Chez 30 malades (47 dosages), le taux moyen est de $127 \mathrm{~g}$ par 1 $(\mathrm{Sm}=3,6)$ et de $123 \mathrm{~g}(\mathrm{Sm}=3,6) \mathrm{chez}$ 42 témoins sains. La différence est non significative d'autant plus que les résultats individuels sont très variables dans les deux groupes (valeurs extrêmes : 85 à 175 chez les malades, 80 à 165 chez les témoins). Il n'existe pas de relation entre ce taux et celui des protéines sériques.

Jeunes bovins. 60 dosages chez les malades établissent le taux moyen à $101 \mathrm{~g}(\mathrm{Sm}=3,4)$ dans les cas graves contre 110 dans les cas légers $(\mathrm{Sm}=2,2)$ et chez les sujets sains $(\mathrm{Sm}=4,2)$. Il y a donc discordance avec les résultats constatés chez les adultes.

\section{- Phosphatases acides :}

Bovins adultes. Chez 36 malades (62 dosages), le nombre d'unités Babson - Read est en moyenne de $1,9(\mathrm{Sm}=0,17$ - v.e. : $0,1-4,3)$ et de $2,2(\mathrm{Sm}=0,15$ - v.e. : $0,5-6,7) \mathrm{chez}$ 43 sujets sains. Différence non significative.

Jeunes bovins. Le nombre moyen d'unités chez les malades (62 dosages) est de 2,2 $(\mathrm{Sm}=0,13)$ dans les cas graves contre 2,3 $(\mathrm{Sm}=0,14)$ dans les cas légers et 1,6 $(\mathrm{Sm}=0,13$ - v.e. : $0,8-2,6)$, chez les sujets sains.

\section{- Phosphatases alcalines:}

Bovins adultes. Chez 37 malades (63 dosages), le nombre moyen d'unités Klein - Babson Read est de 8,3 ( $\mathrm{Sm}=1,23$ ) contre 7,3 $(\mathrm{Sm}=0,92)$ chez 43 sujets sains. La différence est non significative entre ces deux moyennes bien que 16 p. 100 des malades aient des taux supérieurs à 15 unités contre 7 p. 100 seulement des sujets sains.

Jeunes bovins. Le nombre d'unités chez les malades (61 dosages) est de 4,3 dans les cas graves $(\mathrm{Sm}=1,10), 5,2$ dans les cas légers
$(\mathrm{Sm}=0,56)$ contre $4,8(\mathrm{Sm}=0,91) \mathrm{chez}$ les témoins.

\section{- Transaminases :}

Bovins adultes. L'activité de la transaminase glutamique oxaloacétique (T.G.O.) chez $7 \mathrm{ma}$ lades était de 45 unités Frankel $(\mathrm{Sm}=3,1)$ et de 46 unités $(\mathrm{Sm}=0,6)$ chez 28 sujets sains d'abattoir. Différence non significative. L'activité de la transaminase glutamique pyruvique (T.G.P.) était de 40 unités Frankel $(\mathrm{Sm}=1,3)$ chez ces malades et de 45 unités chez les 28 témoins $(\mathrm{Sm}=0,7)$. Il ne semble donc pas que le taux des transaminases soit modifié de façon appréciable.

Jeunes bovins. Dans les cas graves, l'activité de la T.G.O. est de 34 unités $(\mathrm{Sm}=1,5)$ et celle de la T.G.P. de 34 unités également $(\mathrm{Sm}=1,2)$, dans les cas légers 35 unités T.G.O. ( $\mathrm{Sm}=0,8)$ et 36 T.G.P. $(\mathrm{Sm}=0,8)$ et chez les sujets sains 38 unités T.G.O. $(\mathrm{Sm}=1,3)$ et 39 T.G.P. $(\mathrm{Sm}=1,0)$.

\section{- Cholinestérases :}

L'abaissement du pH (méthode de CHARY et collab.) déterminé chez 14 malades était de 1,073 unités (v.e. : 0,848 -1,307) soit un taux d'activité des cholinestérases de 89 p. 100. Mais il est difficile de déterminer si cet abaissement est significatif car l'activité cholinestérasique est profonidément modifiée par les traitements insecticides. C'est ainsi que sur 33 dosages pratiqués sur bovins d'abattoir sains (non traités), l'abaissement du $\mathrm{pH}$ est de 1,287 contre 0,791 dans 75 dosages pratiqués sur des bovins sains traités au carbaryl: or certains animaux atteints de streptothricose avaient subi un tel traitement. Quoi qu'il en soit, s'il y a modification de ces valeurs, elle ne peut être que peu importante.

\section{- Calcium et phosphore :}

Bovins adultes. Chez 19 malades (44 dosages), le taux moyen de calcium total est de $90 \mathrm{mg}$ par $1(\mathrm{Sm}=2,3)$ légèrement inférieur à celui de 34 témoins sains : $95 \mathrm{mg}(\mathrm{Sm}=1,5)$. En ce qui concerne le phosphore minéral, ce taux est de $72 \mathrm{mg}$ par 1 chez 37 malades $(\mathrm{Sm}=2,5)$ contre $64 \mathrm{mg}$ chez 44 sujets sains $(\mathrm{Sm}=2,2)$. Pour ces deux éléments la différence est donc significative et le rapport $\mathrm{Ca} / \mathrm{P}$ est nettement plus bas $(1,25)$ chez les malades que chez les sujets sains $(1,50)$. 
Jeunes bovins. Le taux moyen de calcium total est de $76 \mathrm{mg}(\mathrm{Sm}=2,8)$ dans les cas graves, $79 \mathrm{mg}(\mathrm{Sm}=2,6)$ dans les cas légers et $76 \mathrm{mg}$ chez les témoins $(\mathrm{Sm}=3,4)$. Chez les jeunes, la maladie influe donc peu sur la teneur en calcium, qui est par ailleurs plus basse que chez l'adulte. En ce qui concerne le phosphore minéral, le taux moyen est de $82 \mathrm{mg}(\mathrm{Sm}=3,6)$ dans les cas graves et $74 \mathrm{mg}(\mathrm{Sm}=3,1)$ dans les cas légers alors qu'il est de $89 \mathrm{mg}(\mathrm{Sm}=2,7)$ chez les sujets sains (taux inférieur à celui des adultes). Il n'y a donc pas de relation entre la gravité de la maladie et la teneur du sang en phosphore.

\section{- Bilirubine totale}

Bovins adultes. Chez 12 malades le taux moyen est de $5 \mathrm{mg}$ par $1(\mathrm{Sm}=0,6$ - v.e. : 3 - 10) contre $9 \mathrm{mg}(\mathrm{Sm}=0,8$ - v.e. : 1 - 19) chez 45 sujets sains. Cette différence est significative.

Jeunes bovins. Chez les malades (63 dosages), le taux moyen est de $10 \mathrm{mg}(\mathrm{Sm}=2$ v.e.: 3 -22) dans les cas graves, de $5 \mathrm{mg}$ $(\mathrm{Sm}=0,2$ - v.e. : $3-8)$ dans les cas légers et de $6 \mathrm{mg}(\mathrm{Sm}=0,9$ - v.e. : $4-8) \mathrm{chez}$ les sujets sains. A l'exception d'un cas exceptionnel $(22 \mathrm{mg})$, les taux de bilirubine varient donc peu chez les jeunes malades.

\section{- Fibrinogène}

Bovins adultes. Chez 30 malades (60 dosages), la valeur moyenne est de $6,3 \mathrm{~g}$ par 1 $(\mathrm{Sm}=0,26)$ contre $5,2 \mathrm{~g}(\mathrm{Sm}=0,18)$ chez 42 sujets sains. La différence est hautement significative. Du reste le caillot de sang des sujets sévèrement atteints a fréquemment une consistance très ferme, laissant exsuder peu de sérum. Le taux le plus élevé observé chez les témoins était de 7,99, alors que 5 malades avaient des taux supérieurs (de 8 à $10,6 \mathrm{~g}$ ). Cette augmentation fut très rapide dans un cas grave où elle est passée de $6,25 \mathrm{~g}$ à $11,40 \mathrm{~g}$ en 11 jours. Cependant, cette corrélation directe n'existe pas dans tous les cas : certains malades graves ont un taux normal, ou presque, alors que des sujets sains ont des taux nettement supérieurs à la normale.

Jeunes bovins. 61 dosages sur les malades ont établi le taux moyen de 5,3 $\mathrm{g}(\mathrm{Sm}=0,24)$ dans les cas graves, $5,4 \mathrm{~g}(\mathrm{Sm}=0,16)$ dans les cas légers contre $4,6 \mathrm{~g}(\mathrm{Sm}=0,11)$ chez les témoins. Ce taux est donc plus faible que celui des adultes, mais reste toujours relativement élevé chez les malades.

\section{- Cholestérol :}

Bovins adultes. Chez 35 malades, la valeur móyenne est de $1,28 \mathrm{~g}$ par $1(\mathrm{Sm}=0,48)$, nettement inférieure à celle des 44 témoins : $1,48 \mathrm{~g}(\mathrm{Sm}=0,054)$ avec lesquels il y a donc une différence hautement significative.

Jeunes bovins. Dans les cas graves, le taux moyen est de $0,74 \mathrm{~g}(\mathrm{Sm}=0,055)$ et de $0,87 \mathrm{~g}(\mathrm{Sm}=0,047)$ dans les cas légers. Chez les sujets témoins, le taux normal est de $0,97 \mathrm{~g}(\mathrm{Sm}=0,091)$. Ces résultats confirment ceux de bovins adultes et il semble exister une corrélation entre le taux de cholestérol et la gravité des lésions. Aucun résultat anormalement élevé n'a été noté au cours des 67 dosages effectués sur les malades adultes ou jeunes.

- Index - Tyrosine de la polypeptidémie.

Bovins adultes. Chez 9 malades (17 dosages), la valeur moyenne de l'index est de $31 \mathrm{mg}$ par 1 $(\mathrm{Sm}=1,7-$ v.e. : $22-35$ ) alors que chez 45 témoins sains elle s'élève à $46 \mathrm{mg}$ ( $\mathrm{Sm}=$ 1,8). La différence est hautement significative.

Jeunes bovins. Dans les cas graves, l'index était de $36 \mathrm{mg}(\mathrm{Sm}=1,4)$, dans les cas légers de $34 \mathrm{mg}(\mathrm{Sm}=1,1)$ et chez les témoins sains de $37 \mathrm{mg}(\mathrm{Sm}=1,8)$. Ces valeurs, déterminées au cours de 60 dosages, variaient de 27 à $40 \mathrm{mg}$, étant donc toujours inférieures à celles des adultes.

\section{- Protéinogrammes :}

La plupart des valeurs du protéinogramme subissent des variations très importantes au cours de la streptothricose. Nous avons reporté leur étude globale à la fin de ce chapitre.

Bovins adultes. Les protéinogrammes ont été déterminés sur 41 malades (86 déterminations) et 49 témoins sains.

1. Albumines sériques: Le pourcentage d'albumine par rapport aux protéines totales est de $22,2(\mathrm{Sm}=1,34)$ chez les malades contre $33(\mathrm{Sm}=0,49) \mathrm{chez}$ les témoins. $L a$ différence est hautement significative. Chez les malades, ces taux se répartissent comme suit: 3 à 5 p. $100: 3$ cas -5 à 10 p. $100: 1$ cas 10 à 15 p. $100: 4$ cas -15 à 20 p. 100 : 7 cas -20 à 25 p. $100: 10$ cas -25 à 30 
p. $100: 9$ cas. Plus de 30 p. $100: 7$ cas. Les variations du taux suivent assez fidèlement l'évolution de la maladie, s'abaissant lorsqu'elle progresse (à la limite les taux atteignent 5,4 ou même 3 p. 100) et augmentant lorsqu'elle régresse. Dans certains cas, il est un élément de pronostic sûr: c'est ainsi qu'une génisse, atteinte de streptothricose généralisée mais dont l'aspect physique pouvait laisser espérer une longue résistance à la maladie, avait un taux d'albumine de 7 puis 6 p. 100 à 11 jours d'intervalle : elle mourut quelques jours plus tard. En revanche, chez les animaux peu atteints ou en voie de guérison ce taux est sensiblement normal.

2. Alpha 1 globulines. La proportion de ces globulines est de 5,2 p. $100(\mathrm{Sm}=0,25) \mathrm{chez}$ les malades contre 5,6 p. $100(\mathrm{Sm}=0,18)$ chez les sujets sains: la différence est non significative. Chez 25 malades, ce taux était inférieur à 5,6 p. 100 et chez 16 autres il était supérieur à ce chiffre.

3. Alpha 2 globulines. Leur proportion est de 8 p. $100(\mathrm{Sm}=0,34)$ chez les malades et de $9,5(\mathrm{Sm}=0,26) \mathrm{chez}$ les témoins. La différence est hautement significative. Chez 31 malades, ce taux était inférieur à 9,5 p. 100 et chez les autres il était supérieur à ce chiffre.

4. Béta-globulines. Leur taux est parfois difficile à apprécier avec précision lorsque le taux de gamma-globulines est élevé (limite de séparation peu infléchie). Il est en moyenne de 14,9 p. $100(\mathrm{Sm}=0,51)$ chez les malades contre 14 p. $100(\mathrm{Sm}=0,29)$ chez les sujets sains. La différence est non significative. Chez les malades, 17 taux étaient inférieurs à $14 \mathrm{p}$. 100 et 21 taux supérieurs à ce chiffre.

5. Gamma-globulines. Leur taux subit des variations très importantes, inverses de celles des albumines. Chez les malades, il était de 49,7 p. $100(\mathrm{Sm}=1,49)$ contre 37,9 p. 100 $(\mathrm{Sm}=0,65) \mathrm{chez}$ les témoins. Cette différence est hautement significative et elle peut atteindre des valeurs très élevées (dans un cas : $73 \mathrm{p}$. 100). C'est ainsi que chez les 41 malades, si 2 sujets avaient un taux inférieur à 38 p. 100 , 20 avaient un taux de 38 à 50 p. 100,14 un taux de 50 à 60 p. 100 et 5 un taux supérieur à 60 p. 100 .

Afin de contrôler si, outre les modifications quantitatives du protéinogramme, n'existaient pas des modifications qualitatives de ses diffé- rentes fractions, nous avons effectué l'analyse immuno-électrophorétique de 25 sérums de zébus atteints de streptothricose grave. L'analyse a été réalisée selon les techniques classiques détaillées par A.J. CROWLE (6) à l'aide d'un sérum antibovin élaboré par des lapins hyperimmunisés avec un mélange de trois sérums de bovins sains.

En comparant toujours les résultats obtenus avec ceux de sérums témoins prélevés sur des sujets sains, il ne nous a jamais été donné de constater de différence notable constante entre les différentes fractions hormis les différences quantitatives déjà décelées par l'électrophorèse. Il n'y a pas de déplacement ni d'apparition de lignes de précipitations protéiniques visibles au cours de la streptothricose, du moins avec les techniques utilisées (photo 1).

6. Rapport Albumines/Globulines. Du fait de la baisse des albumines et de l'augmentation des globulines, ce rapport est abaissé au cours de la maladie : $0,30 \mathrm{chez}$ les malades $(\mathrm{Sm}=$ $0,02)$ contre 0,50 chez les sujets sains $(\mathrm{Sm}=$ 0,01 ). Dans les cas les plus graves, ce rapport atteint des valeurs très basses : $0,04-0,05$ $0,05-0,07$ chez quatre sujets très atteints.

La répartition des valeurs dans le groupe des malades est la suivante: 0,04 à 0,10 : 4 animaux - 0,10 à $0,20: 7$ animaux 0,20 à $0,30: 10$ animaux $-0,30$ à 0,40 : 9 animaux $-0,40$ à $0,50: 8$ animaux. Supérieur à $0,50: 3$ animaux.

Afin d'illustrer cette évolution, nous reproduisons ci-dessous les dosages d'albumines et globulines réalisés sur quatre animaux malades dont deux ont vu leur état aggravé et deux ont guéri spontanément.

Premier cas: Vache métis Normande. Atteinte extrêmement sévère de streptothricose généralisée, état cachectique : l'animal meurt quelques heures après le dernier prélèvement. On constate un taux extrêmement bas des albumines et les gamma-globulines ont une valeur très élevée (Tableau $n^{*}$ II).

Second cas: Vache 1/2 Brahman. Atteinte sévère de streptothricose en voie d'aggravation. Etat général médiocre: le taux d'albumines sériques s'abaisse progressivement alors que les gamma-globulines augmentent (Tableau $\mathbf{n}^{\circ}$ III).

Troisième cas: Taureau 3/4 Brahman. Atteinte sévère de streptothricose qui a évolué 
IABLEAU $\mathrm{N}^{\circ}$ II

\begin{tabular}{|l|c|c|c|c|c|}
\hline D a t e s & $24 / 7$ & $3 / 8$ & $18 / 8$ & $28 / 8$ & $4 / 9$ \\
\hline Albumines (p.100) & 10 & 6 & 3 & 3 & 3 \\
\hline $\begin{array}{l}\text { Gamma-Globulines } \\
(p .100)\end{array}$ & 66 & 69 & 73 & 71 & 72 \\
\hline Rapport $\frac{A}{G}$ & 0,11 & 0,06 & 0,03 & 0,03 & 0,03 \\
\hline
\end{tabular}

TABLEAU $N^{\circ}$ III

\begin{tabular}{|l|l|l|l|l|l|l|l|l|}
\hline D a t e s & $11 / 6$ & $4 / 7$ & $1 / 8$ & $13 / 8$ & $27 / 8$ & $11 / 9$ & $27 / 9$ & $4 / 11$ \\
\hline Albumines (p.100) & 29 & 27 & 26 & 23 & 23 & 23 & 22 & 19 \\
\hline $\begin{array}{l}\text { Gamma-Globulines } \\
\text { (p.100) }\end{array}$ & 40 & 43 & 44 & 48 & 46 & 48 & 48 & 55 \\
\hline Rapport $\frac{\mathrm{A}}{\mathrm{G}}$ & 0,41 & 0,37 & 0,35 & 0,30 & 0,30 & 0,30 & 0,28 & 0,23 \\
\hline
\end{tabular}

lentement jusqu'à la $2^{\circ}$ quinzaine de décembre à peu. Le taux des albumines qui s'était abaissé avec un état général médiocre. A partir de ce progressivement tend à s'améliorer faiblement moment, la maladie a régressé lentement et à partir de cette époque mais le taux des spontanément et l'état général s'améliore peu gamma-globulines reste élevé (Tableau $\mathbf{n}^{\circ}$ IV).

TABLEAU $N^{\circ}$ IV

\begin{tabular}{|c|l|l|l|l|l|l|l|l|l|l|}
\hline Da te s & $6 / 10$ & $6 / 11$ & $14 / 11$ & $20 / 11$ & $27 / 11$ & $7 / 12$ & $21 / 12$ & $2 / 1$ & $24 / 1$ & $26 / 2$ \\
\hline Albumines (p.100 & 26 & 23 & 22 & 20 & 19 & 19 & 19 & 20 & 22 & 22 \\
\hline $\begin{array}{l}\text { Gamma-Globulines } \\
\text { (p.100) }\end{array}$ & 53 & 56 & 54 & 56 & 54 & 55 & 55 & 52 & 56 & 53 \\
\hline Rapport $\frac{\mathrm{A}}{\mathrm{G}}$ & 0,35 & 0,30 & 0,28 & 0,25 & 0,23 & 0,23 & 0,23 & 0,25 & 0,28 & 0,28 \\
\hline
\end{tabular}

Quatrième cas: Taureau $3 / 4$ Brahman. faible remontée du taux des albumines qui Atteinte légère de streptothricose évoluant avaient conservé une valeur sub-normale et spontanément vers la guérison. Bon état géné- surtout un net abaissement du taux des gammaral. On constate, à partir de mi-novembre, une globulines (Tableau $n^{*}$ V).

TABLEAC $\mathrm{N}^{\circ} \mathrm{V}$

\begin{tabular}{|l|l|l|l|l|l|l|l|l|l|}
\hline \multicolumn{1}{|c|}{ D a t e s } & $6 / 10$ & $6 / 11$ & $14 / 11$ & $20 / 11$ & $27 / 11$ & $7 / 12$ & $21 / 12$ & $2 / 1$ & $24 / 1$ \\
\hline Albumines (p.100) & 33 & 29 & 29 & 30 & 32 & 31 & 34 & 33 & 35 \\
\hline $\begin{array}{l}\text { Gamma-Globulines } \\
(\mathrm{p} .100)\end{array}$ & 41 & 48 & 46 & 41 & 43 & 43 & 37 & 35 & 35 \\
\hline Rapport $\frac{\mathrm{A}}{\mathrm{G}}$ & 0,49 & 0,41 & 0,41 & 0,43 & 0,47 & 0,45 & 0,51 & 0,49 & 0,54 \\
\hline
\end{tabular}


Jeunes bovins. 60 déterminations ont été faites sur les 22 malades avec les résultats suivants :

TABLEAU $N^{\circ} \mathrm{VI}$

\begin{tabular}{|c|c|c|c|c|c|c|}
\hline \multicolumn{3}{|c|}{ Cas graves } & \multicolumn{2}{|c|}{ Cas légers } & \multicolumn{2}{|r|}{ Sujets sains } \\
\hline Albumines & $: 25,5 \mathrm{p} .100$ & $(\mathrm{Sm}=1,88)$ & $32,5 \mathrm{p}, 10$ & $(\mathrm{Sm}=1,15)$ & 30,5 & $\mathrm{p} .100(\mathrm{Sm}=0,85)$ \\
\hline A1pha 1-Globulines & $: \quad 6,2 \mathrm{p} \cdot 100$ & $(\mathrm{Sm}=0,44)$ & $6,5 \mathrm{p} \cdot 10$ & $(\mathrm{Sm}=0,26)$ & 6,2 & $\mathrm{p} .100(\operatorname{Sin}=0,34)$ \\
\hline Alpha 2-Globulines & : $8,2 \mathrm{p} \cdot 100$ & $(S m=0,41)$ & 9,4 p. 10 & $($ Sm $=0,2 B)$ & 10,1 & $\mathrm{p} .100(\mathrm{Sm}=0,57)$ \\
\hline Béta-Globulines & $: 43,9$ p. 100 & $(\mathrm{Sm}=1,76)$ & 13,5 p. 10 & $(\mathrm{Sm}=0,44)$ & 14,5 & p. $100\left(S_{i n}=0,59\right)$ \\
\hline Gamma-Globu1ines & $: 46,2 \mathrm{p} .100$ & $(\mathrm{Sm}=1,78)$ & $38,1 \mathrm{p} \cdot 10$ & $\left(S_{m}=0,91\right)$ & 38,7 & p.100 $(\mathrm{Sm}=1,44)$ \\
\hline Rapport $\frac{A}{G}$ & 0,35 & $(\mathrm{Sm}=0,033)$ & $0,49 \mathrm{p} \cdot 10$ & $(\mathrm{Sm}=0,024)$ & 0,44 & $p \cdot 100(\mathrm{Sm}=0,017)$ \\
\hline
\end{tabular}

Chez ces jeunes animaux, le taux d'albumine est donc également d'autant plus bas que la maladie est plus grave comme le démontre la concordance entre le classement des animaux selon l'atteinte clinique et le dosage de leurs albumines :

- Traces de streptothricose (10 animaux): Albumine : 32 p. 100;

- Atteinte légère (4 animaux):

Albumine : 27 p. 100;

- Atteinte nette (6 animaux):

Albumine : 25 p. 100;

- Atteinte grave (2 animaux):

Albumine : 22 p. 100.

Pendant la période de 3 mois, au cours de laquelle ces animaux ont été mis en observation, la maladie a évolué chez certains d'entre eux. Toutes les fois qu'une amélioration clinique de la maladie a été constatée, nous avons noté un relèvement des taux d'albumine. De même lorsqu'une aggravation a été constatée, elle était accompagnée d'un abaissement de ce taux : sur deux sujets morts de la maladie le taux a baissé à 17 p. 100 et 18 p. 100 dans les jours précédents.

En ce qui concerne les Alpha et Béta-globulines, les modifications sont peu sensibles au cours de la maladie.

Par contre, les gamma-globulines sont nettement augmentées, quoique de façon moins importante que chez les adultes. C'est ainsi que, chez les sujets sains, ce taux varie de 36 à 42 p. 100 contre 32 à 43 p. 100 chez les sujets légèrement atteints et 41 à 55 p. 100 chez les sujets gravement atteints.
En résumé, l'étude des protéinogrammes des bovins atteints de streptothricose, et plus particulièrement ceux des bovins adultes, nous semble revêtir une importance particulière. Ces protéinogrammes sont caractérisés d'une façon générale :

1. Par une baisse du taux des albumines, qui peut être très importante dans les cas graves;

2. Par une baisse légère des Alpha-globulines;

3. Par une légère élévation du taux des Béta-globulines;

4. Par une augmentation du taux des Gamma-globulines qui peut être très importante dans les cas graves.

De plus, les variations du taux d'albumine paraissent liées étroitement à l'évolution de la maladie et à l'état général. Nous avons souvent constaté que le taux des albumines sériques pouvait être utilisé, pour établir un pronostic, avec beaucoup plus de certitude que ne peut le faire le simple examen clinique de l'animal.

De même, nous avons pu constater que l'importance de relèvement du taux des albumines après un traitement aux antibiotiques (3) pouvait permettre d'apprécier, de façon précoce, l'efficacité du traitement, ou la nécessité de procéder à son renouvellement.

\section{ETUDE DE L'EVOLUTION DES ANTICORPS}

\section{AU COURS DE LA STREPTOTHRICOSE NATURELLE}

Afin de déterminer l'évolution des anticorps 
circulant chez les animaux malades, des prises de sang ont été effectuées sur des animaux à divers stades de la maladie, soit que celle-ci évolue naturellement, soit qu'elle soit arrêtée par un traitement aux antibiotiques.

\section{METHODES}

Les sérums sont prélevés selon la technique classique et congelés à $-30^{\circ}$. L'étude complète d'un lot sera effectuée en une seule opération afin d'éviter les décongélations successives. Nous n'avons utilisé en pratique que deux méthodes d'étude des anticorps: Précipitodiffusion en gélose (pour sa commodité d'exécution) et hémagglutination passive (pour sa plus grande sensibilité et ses résultats quantitatifs) selon les méthodes décrites par PERREAU et CHAMBRON (13) puis PULLIAM, KELLEY et COLES (15).

Dans le cas de la précipitation-diffusion en gélose, nous avons utilisé un antigène brut constitué par le surnageant d'une culture de trois mois (en bouillon-tryptose "DIFCO " de Dermatophilus congolensis lyophilisé puis repris au $1 / 10$ de son volume initial selon la technique de VIGIER et BALIS (18).

Dans le cas de l'hémagglutination passive, nous avons utilisé l'antigène polyosidique puri- fié, extrait selon la méthode de WESTPHAL et LUDERITZ, pour sensibiliser des hématies fraîches de mouton.

\section{RESULTATS}

Ils concernent deux groupes de malades: celui des animaux adultes, atteints de maladie naturelle et vivant en élevage extensif et celui des jeunes animaux ( 8 à 12 mois) contaminés naturellement puis gardés en observation à l'étable. L'évolution des lésions chez eux a été celle des autres sujets témoins du troupeau durant la période considérée (fin de saison des pluies et saison sèche): guérisons spontanées progressives dans la plupart des cas, aggravation et mort dans quatre cas.

\section{A. Résultats de l'analyse par précipitation-diffusion en gélose}

1. Sur 21 sujets élevés à l'étable ayant guéri de cette maladie, 11 présentaient des anticorps précipitants dès le premier examen. Ces sérums sont restés précipitants pendant huit mois, sauf chez un animal guéri. Des précipitines sont apparues dans le sérum de 3 animaux au cours du même temps, les 7 autres n'en ont jamais présentées.

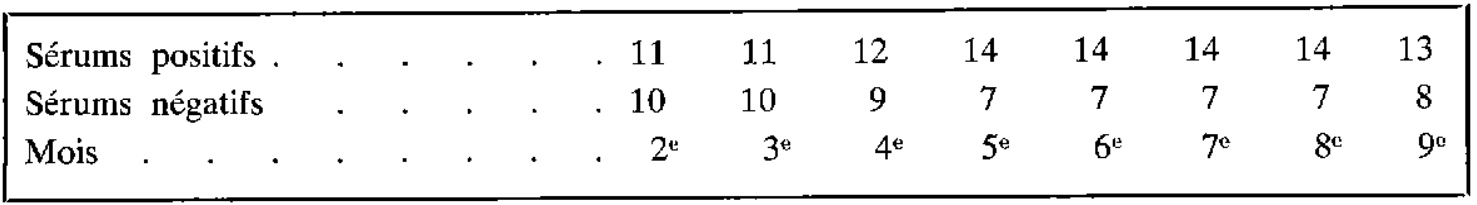

Les premières lésions n'ayant été observées chez ces animaux que vers l'âge de 6 mois, il est possible de conclure que, Jorsqu'ils existent, les anticorps précipitants apparaissent chez la majorité des sujets entre le $1^{\text {er }}$ et le $6^{\mathrm{e}}$ mois de la maladie et qu ils persistent au moins jusqu'à la fin de la maladie. Les 4 sujets élevés à l'étable, et morts de leur maladie ont présenté des anticorps précipitants jusqu'à leur mort.

2. Sur les 65 sujets élevés en liberté : 36 présentaient des anticorps précipitants, sans qu'il soit possible de préciser leur date d'apparition ni leur évolution ultérieure.

\section{B. Résultats de l'analyse par la méthode de I'hémagglutination passive}

1. Sur les 21 sujets élevés à l'étable et guéris de leur maladie, le taux initial d'anticorps était très différent selon les animaux :
$1 / 80$ : 2 animaux;
$1 / 320$ : 6 animaux;
$1 / 1.280: 2$ animaux;
$1 / 160$ : 6 animaux;
$1 / 640$ : 4 animaux;
$1 / 2.560: 1$ animal.

L'évolution de ce taux a été également variable: il a augmenté chez deux animaux, 
diminué chez quinze, et est resté identique chez les quatre sujets qui devaient mourir de leur maladie. Si l'on ne prend en considération que le groupe majoritaire des quinze animaux, l'évolution des anticorps peut être ainsi schématisée au cours du temps:

- $1^{\text {er }}$ au $3^{\text {e }}$ mois de la maladie: le taux d'anticorps varie de $1 / 1.280$ à $1 / 160$.

$-4^{\mathrm{e}}$ au $6^{\mathrm{e}}$ mois de la maladie : le taux d'anticorps varie de $1 / 640$ à $1 / 160$.

- $7^{\mathrm{o}}$ au $9^{\mathrm{e}}$ mois de la maladie: le taux d'anticorps varie de $1 / 640$ à $1 / 80$.

2. Sur les 65 sujets élevés en liberté, nous n'avons effectué que deux titrages, à un mois d'intervalle :

a) Premier titrage : le taux varie de $1 / 40$ à $1 / 2.560$;

b) Second titrage :

- Chez 30 animaux malades, non traités : Titre identique dans 8 cas, titre abaissé dans 19 cas, titre augmenté dans 3 cas.

- Chez 35 animaux malades traités 15 jours auparavant par des antibiotiques: Titre identique dans 4 cas, titre abaissé dans 6 cas, titre augmenté dans 25 cas.

Il existe donc une augmentation statistiquement significative du taux des anticorps à la suite d'un traitement aux antibiotiques (3).

\section{ETUDE HISTOLOGIQUE DES LESIONS ORGANIQUES DANS LA STREPTOTHRICOSE NATURELLE}

Des fragments d'organes de bovins morts de streptothricose naturelle ont été prélevés aux fins d'examen histologique des lésions.

Les résultats d'ensemble de 62 analyses concernant 22 animaux peuvent être ainsi schématisés :

Foie: Présence de lésions graves dans 9 analyses sur 15. Reins: 9 lésions graves sur 13 analyses. Rate et ganglions : 5 lésions graves sur 7 analyses. Peau: 8 lésions graves sur 10 analyses. Testicules: Absence de lésions.

L'examen des coupes permet de classer les lésions en deux groupes: d'une part, celles résultant de l'action directe du germe et qui sont des lésions inflammatoires localisées au niveau de la peau, puis à échéance secondaire des réactions du système lymphatique (rate et ganglions); d'autre part, des lésions d'intoxication localisées principalement au niveau du foie et des reins.

En ce qui concerne les lésions dues à une intoxication, faut-il incriminer une substance toxique provenant du germe lui-même ou bien ces lésions sont-elles le résultat d'une autointoxication consécutive au profond délabrement de l'organisme à la suite d'une longue et épuisante maladie? Histologiquement, la deuxième hypothèse paraît plus vraisemblable, les lésions observées au niveau du foie et des reins étant aiguës ou subaiguës mais n'atteignant pour ainsi dire jamais le stade de la chronicité. Cela confirmerait les échecs rencontrés dans la recherche d'une toxine éventuelle de Dermatophilus congolensis (13).

La peau: Les lésions cutanées ont été décrites d'une façon détaillée par G. THIERY (12) et nos observations ne font que confirmer les siennes. L'épiderme montre, selon le stade de ces lésions, soit une hyperkératose (photo 2), soit un véritable nivellement qui peut aller jusqu'au stratum granulosum ou même jusqu'au derme. Dans tous les cas, on note un allongement des papilles dermiques, une légère turgescence du corps muqueux de Malpighi et une importante accumulation de pigments mélaniques qui se prolonge vers la surface. On peut aussi observer la présence de polynucléaires plus ou moins accumulés entre les strates de la couche cornée ainsi que d'importantes colonies microbiennes surtout localisées vers la surface. Le derme, quant à lui, est peu modifié dans ses couches profondes; on observe une légère dilacération du chorion et quelques légers infiltrats périvasculaires. Dans la couche réticulaire, la réaction inflammatoire est plus importante, caractérisée par d'importants infiltrats cellulaires, constitués essentiellement par des plasmocytes et des histiocytes dérivant tous des histiocytes périvasculaires.

Les ganglions lymphatiques: Ils présentent une hyperactivité notable. On remarque, en particulier, une hyperplasie du tissu réticulaire accompagné d'un épuisement lymphoïde (photo 3). Les sinus sont encombrés de cellules inflammatoires parmi lesquelles prédominent 
les macrophages et les plasmocytes. De place en place, on distingue quelques polynucléaires. Dans certaines zones, on trouve des cellules en voie de dégénérescence et même des débris cellulaires. Dans leur ensemble, tous les sinus sont encombrés par des pigments ferrugineux. Cette hyperplasie atteint non seulement les ganglions drainant les zones périphériques mais se rencontre également dans des ganglions profonds et notamment au niveau des mésentériques, ce qui tendrait à confirmer que l'ensemble du système lymphatique est mobilisé chez les malades gravement atteints.

La rate: Elle est également très souvent réactionnelle. Comme dans les ganglions, on note une métaplasie du tissu réticulaire, caractérisée par une prolifération de cellules macrophagiques. Parfois, il est possible d'apercevoir quelques rares mégaryocytes. On trouve également des dépôts de pigments ferrugineux en abondance, tant libres qu'à l'intérieur des macrophages.

Le foie: Il présente en général des réactions marquées indiquant une intoxication (photo 4). En particulier, on remarque une dilatation des travées hépatiques, accompagnée de phénomènes de dégénérescence granulo-graisseuse d'un certain nombre de cellules hépatiques notamment au niveau des veines centro-lobulaires. Dans certains cas, on observe également des dépôts de pigments ferrugineux et une légère infiltration par des cellules de la lignée lymphoïde. Dans d'autres cas, il est possible d'apercevoir une réaction scléreuse au niveau des espaces portes. Un seul cas de cirrhose avancée a pu être observé. Dans l'ensemble donc, les lésions sont d'ordre toxique mais elles ne semblent pas primitives. Cette impression est confirmée par l'examen des prélèvements de reins.

Les poumons: Les rares examens qui ont pu en être faits ne révèlent rien de particulier si ce n'est, dans quelques cas, une alvéolite de type cedémateux qui semble être surtout en rapport avec une stase d'origine circulatoire ou des phénomènes asphyxiques liés à l'agonie des malades.

Les reins: Ils présentent en général une atteinte de la zone glomérulaire caractérisée parfois par une dilatation des capillaires glomérulaires mais surtout par des lésions dégénératives au niveau des tubes contournés. Les cellules endothéliales tapissant les parois de ces tubes sont atteintes de nécrose et il n'est pas rare d'en trouver dans la lumière des tubes. On observe aussi que les cellules survivantes font un effort important de régénération et les tubes sont alors bordés par des cellules aplaties et très allongées (photo 5).

Il faut souligner que tous les prélèvements ont été effectués sur des animaux au dernier stade d'épuisement et morts de la maladie naturelle. Il resterait à déterminer à quel stade de la maladie ces lésions apparaissent puis deviennent irréversibles, car dès leur installation le pronostic peut être considéré comme fatal et tout traitement illusoire. Dans le cadre d'une prophylaxie sanitaire, de tels sujets devraient être immédiatement éliminés.

\section{INTERPRETATION DES RESULTATS ET CONCLUSION}

L'étude biochimique a révélé des modifications de certains constituants du sang, en particulier cholestérol, fibrinogène, calcium, phosphore, protides sanguins. L'abaissement du taux du cholestérol constaté chez les animaux malades n'est, a priori, pas surprenant, puisqu'il s'observe fréquemment au cours d'infections aiguës graves. Il pourrait peut-être s'expliquer également en partie comme une conséquence de l'hyperactivité du système réticulo-endothélial. Le taux du fibrinogène augmente chez les animaux atteints de streptothricose, comme il augmente dans la plupart des infections localisées ou généralisées, et en particulier dans le cas d'inflammations aiguës et chroniques.

Signalons que cette perturbation se produit en sens inverse de celle qu'on observe souvent dans des maladies où la cellule hépatique est atteinte (cirrhose ...). Dans ces cas, le fibrinogène est au contraire abaissé. L'augmentation du taux de phosphore pourrait être une conséquence d'une atteinte rénale, décelée, du point de vue chimique, par la présence fréquente d'albumine dans les urines de bovins atteints de streptothricose que nous avons analysées. L'abaissement du calcium serait en corrélation avec l'augmentation du phosphore. L'importante diminution du taux d'albumine sérique, constatée dans la streptothricose, s'observe également de façon fréquente dans les maladies infectieuses. Il ne nous semble pas qu'elle puisse 
être expliquée par la seule déperdition urinaire. $\mathrm{Si}$, en effet, nous avons constaté la présence d'albumine dans les urines d'une quinzaine d'animaux atteints de streptothricose, accompagnée le plus souvent de pseudo-albumine, nous n'avons trouvé que des teneurs allant de quelques traces à $2,40 \mathrm{~g}$ par 1 d'urine chez des animaux très gravement atteints. En raison du nombre restreint d'examens d'urines pratiqués, il ne nous est pas possible de généraliser. Il semble cependant plus vraisemblable que la diminution des albumines sériques soit due à une détérioration de fonctions hépatiques, ainsi qu'à une réaction compensatrice d'équilibre due à la forte augmentation des gammaglobulines. Ce dernier mécanisme pourrait également expliquer les irrégularités des résultats successifs des teneurs en protides totaux chez un même animal. Il est à signaler que les Gamma-globulines qui, d'une façon générale, ont tendance à diminuer dans les cas d'inflammation importante ou de destruction tissulaire, sont, au contraire, augmentées dans la streptothricose.

En conclusion, il semble que les modifi- cations des constituants chimiques du sang que nous avons constatées chez les bovins atteints de streptothricose proviennent de lésions hépatiques et rénales, ainsi que d'un hyperfonctionnement des mécanismes de synthèse des globulines.

En effet, l'étude histologique confirme que les lésions inflammatoires, et leurs réactions associées, ne sont pas les principales comme l'avait constaté G. THIERY (17). Des lésions, d'auto-intoxication probablement, atteignent et détruisent les tissus rénal et hépatique. $\mathrm{Ce}$ dernier, en particulier, ne fonctionnant plus, surviennent des bouleversements profonds dans ses synthèses et dans l'équilibre de protides sanguins. Malgré une mobilisation soutenue de ces protides pour la synthèse des gamma-globulines anticorps, l'organisme n'est pas toujours maître d'une infection cutanée qui se généralise.

L'étude successive des constituants du sang, des anticorps sériques et des lésions anatomo-pathologiques contribue à souligner le très grave retentissement de cette maladie " externe" sur l'organisme tout entier.

\section{SUMMARY}

\section{Contribution to the serological and physio-pathological study of bovine streptothricosis}

The biochemical study of the blood of cattle infected with streptothricosis reveals some important modification in its composition, particularly an increase of fibrinogen, a fall of cholesterol, a falling off of ratio $\mathrm{Ca} / \mathbf{P}$ and some deep quantitative perturbation of its proteinic components. These modifications closely follow the evolution of the disease and may allowed us to establish prognosis on it.

The serological study proves also a concordance between the evolution of the lesions and the rate of circulating antibodies.

The histological study of organs of infected cattle dead of natural disease reveals two types of principal lesions: inflammatory lesions, due to the direct action of organism on the skin, spleen and lymphatic nods, and toxical lesions localised in the liver and in the kidney.

\section{RESUMEN}

\section{Contribución al estudio serologico y fisiopatologico de Ia estreptotricosis de los bovinos}

El estudio bioquimico de la sangre de bovinos atacados por la estreptotricosis revela importantes modificaciones de su composición, particularmente un aumento del fibrinógeno, una disminución del colesterol, una baja de la razón $\mathrm{Ca} / \mathbf{P}$ e importantes trastornos de sus constituyentes protidicos: Estas modificaciones estrechamente siguen la evolución de la enfermedad y pueden permitir el establecimiento del pronóstico.

El estudio serológico demuestra igualmente una concordancia entre la evolución de las lesiones y la tasa de anticuerpos circulantes.

El estudio histo-patologico de los órganos de bovinos muertos de la enfermedad natural muestra dos tipos de lesiones principales: jnflamatorias (causadas por la acción directa de la bacteria sobre la piel, el bazo y los ganglios) y tóxicos, localizadas en el higado y en el riñon. 


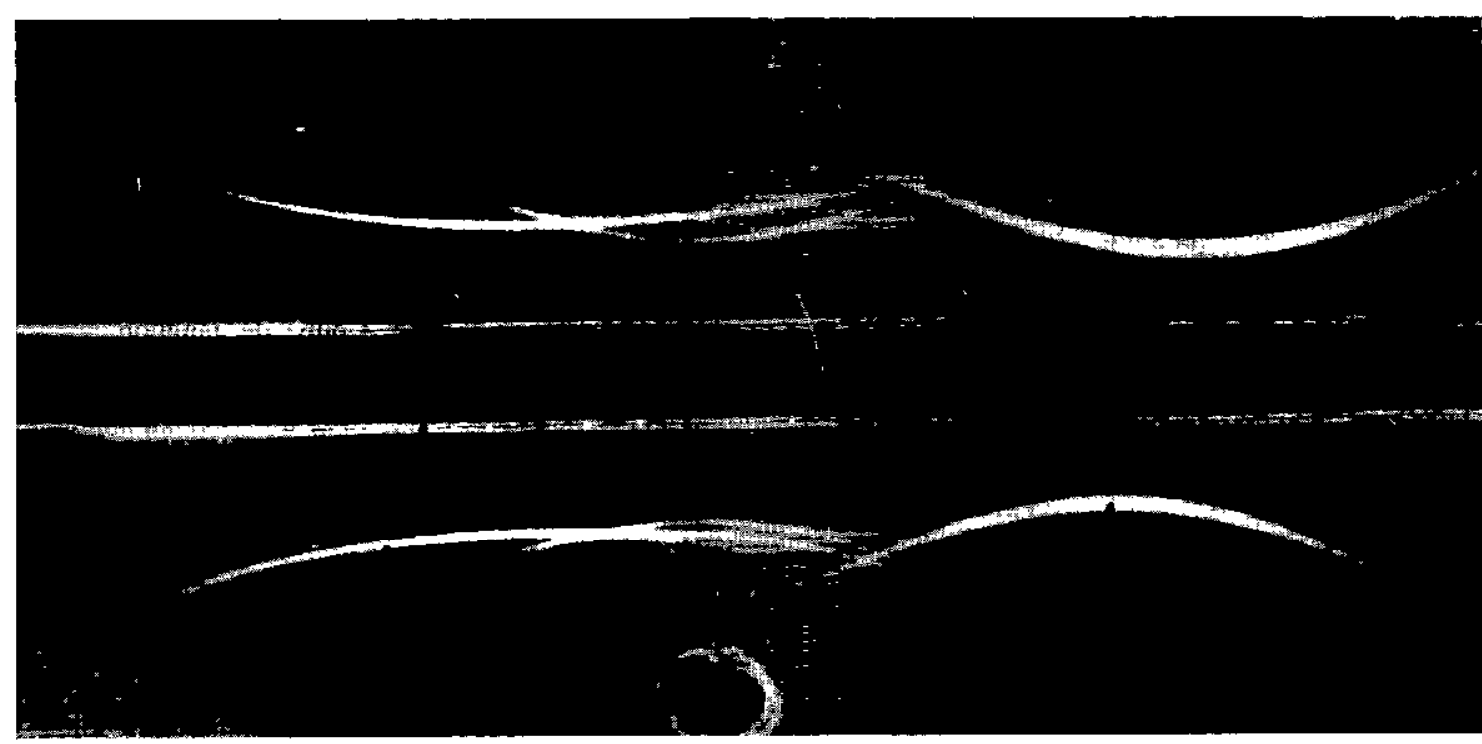

Photo 1. - Analyse immuno-électrophorétique de sérums.

$M=$ Sérum de bovin atteint de streptothricose.

$\mathrm{S}=$ Sérum de bovin sain.

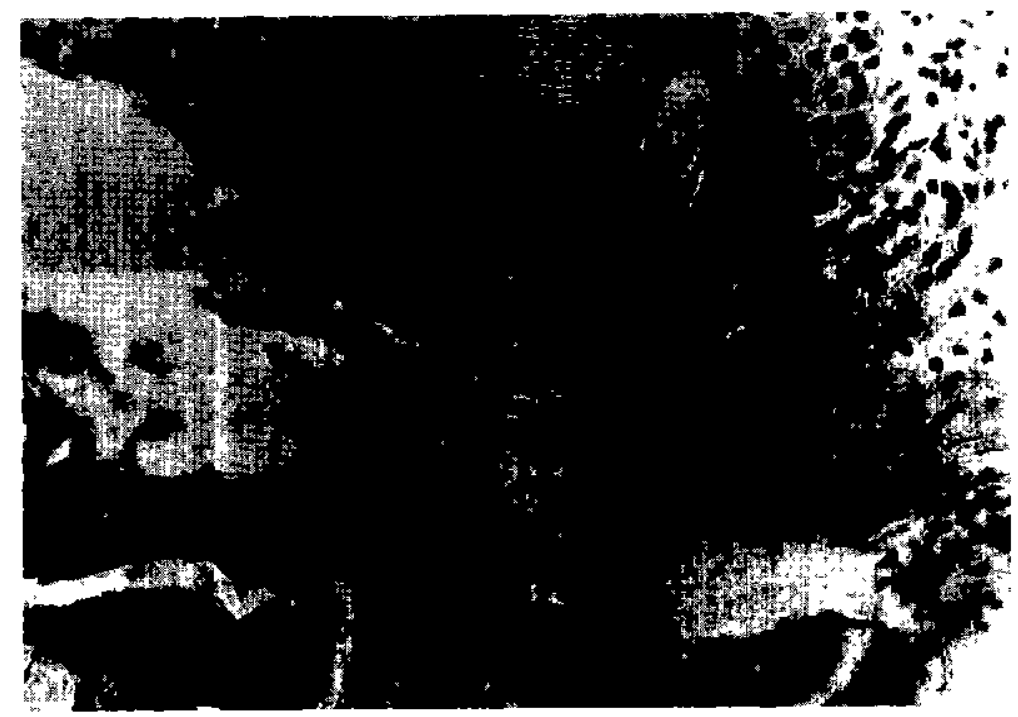

Photo $2-$ Coupe de peau : hyperkératose et desquamation. 


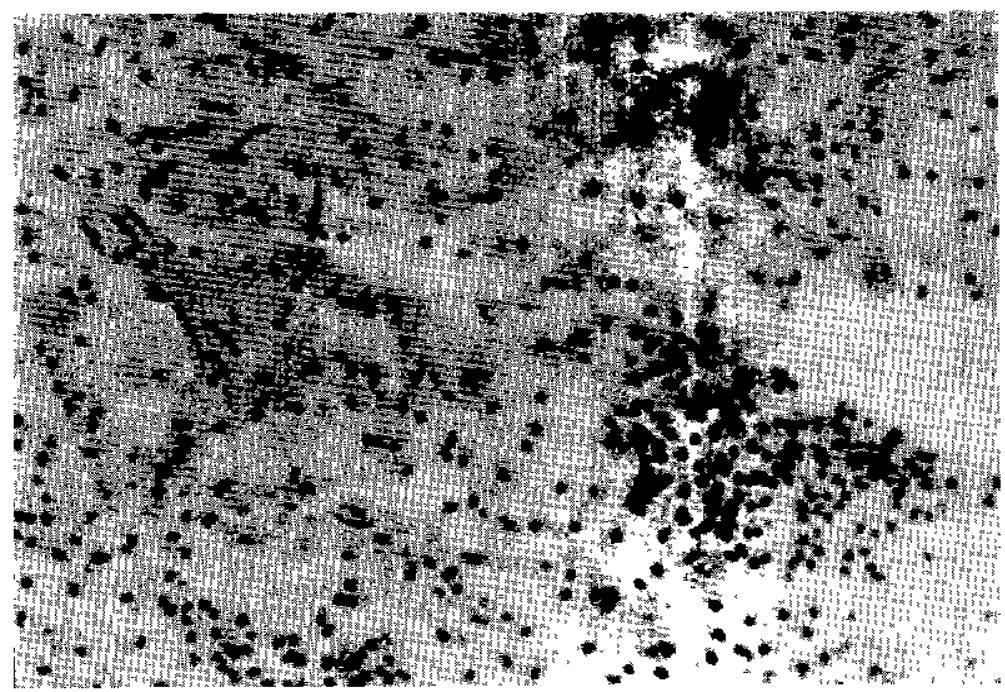

Photo 3.

Coupe de ganglion préscapulaire :

hyperplasie du tissu réticulaire et épuisement lymphoïde.

Photo 4:

Coupe de foie :

réaction d'intoxication

avec nécrose centro-lobulaire.
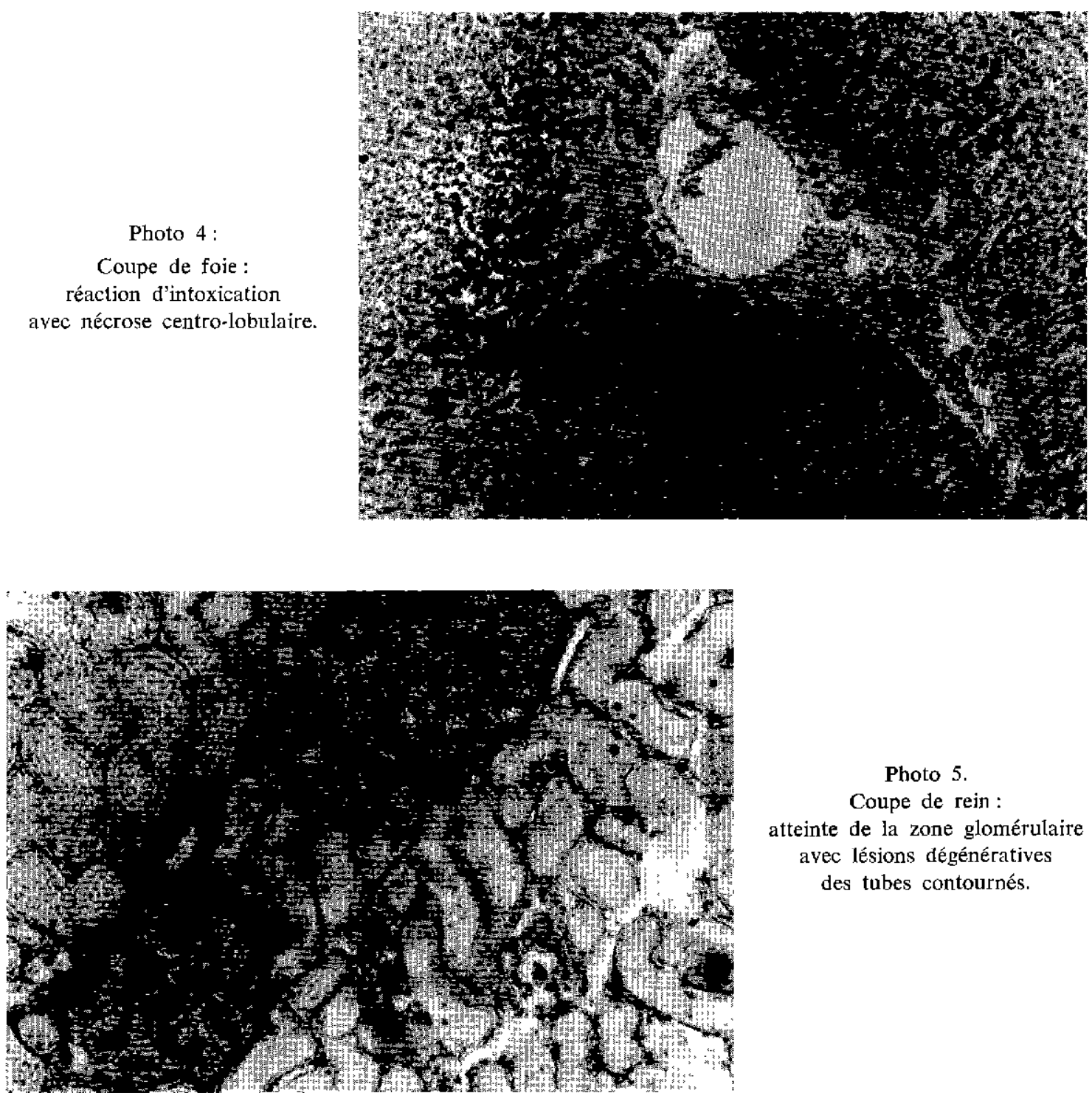

Photo 5 .

Coupe de rein :

atteinte de la zone glomérulaire avec lésions dégénératives des tubes contournés. 


\section{BIBLIOGRAPHIE}

1. BABSON (A. L.), READ (P.A.) et PHILLIPS (C. E.), Am. J. Clin. Path., 1959, $32: 1$.

2. BAILLY (M.), FONTY (P.) et LEGER (N.), Le dosage de l'urée dans les liquides biologiques à l'aide du para-diméthylamine-benzaldéhyde, $A n n$. Biol. Clin., 1967, 25 (10) : 1221-32.

3. BLANCOU (J. M.), Traitement de la streptothricose bovine par une injection unique d'antibiotiques à haute dose. Rev'. Elev. Méd. vét. Pays trop., 1969, 22 (1) : 33-40.

4. BOROVICZENY (K.G. von), Erythrocymetrie Methods and their Standardization. Bâte, S. Karger, 1964.

5. CHARY (R.), JAYOT (R.) et BOCQUET (P.), Contribution à la toxicologie du bétail : titrage de l'activité cholinestérasique sanguine des bovins, Bull. Acad. vét., 1961, 34 ; 167-74.

6. CROWLE (A. J.), Immunodiffusion. New York et Londres, Academic Press, 1961.

7. FINETTI (P.) et BUFFARD (G.), Dosage spécifique du glucose par l'Ortho-toluidine. Extraits des Feuillets de Biologie, 1965, 6 (27) : 59-62.

8. FLEURY (P.), COURTOIS (J.E.) et LECLERC (M.), Fiches techniques de Chrmie Biologique. Paris, Vega, 1965.

9. KLEIN (B.), READ (P. A.) et BABSON (A. L.), Clin. Chem. 8, 1960.
10. LECOCQ (R.), Manuel d'analyses médicales et de biologie clinique. Paris, Doin, 1967.

11. LOISELEUR (J.) et collab., Techniques de Laboratoire. Paris, Masson, 1963.

12. MORNET (P.) et THIERY (G.), Streptothricose cutanée des bovins, Bull. Epiz. Dis. Afr. 1955, 3 (3) : 302-24.

13. PERREAU (P.) et CHAMBRON (J.), Immunologie de la streptothricose cutanée des bovins. Essai de vaccination, Rev. Elev. Méd. v'ét. Pays trop. 1966, 19 (3) : 263-74.

14. PERREAU (P.), Le pouvoir pathogène de Dermatophilus congolensis est-i] lié à la diffusion d'une toxine? Rev. Elev. Méd. l'ét. Pays trop., 1968, 21 : 59-69.

15. PULLIAN (J. D.), KELLEY (D. C.) et COLES (E. H.), Immunologie studies of natural and experimental cutaneous streptothricosis infections in cattle, Am. J. ver. Res. 1967, 28 (123) : 447-55.

16. REITMAN (S.) et FRANKEL (F.), Am. J. Clin. Path., 1957, 28 : 56.

17. THIERY (G.), Streptothricose bovine. Rapport annuel du Laboratoire de l'Elevage de Dakar, 1964.

18. VIGIER (M.) et BALIS (J.), Variabilité et antigénicité de Dermatophilus congolensis, Rev. Elev. Méd. vét. Pays trop. 1967. 20 (1): 67-76. 